\title{
LIRAGLUTIDE IMPROVES SURFACTANT PROTEIN-B PRODUCTION AND REDUCES RIGHT VENTRICULAR HYPERTROPHY IN A RAT MODEL OF INTERSTITIAL PULMONAR FIBROSIS
}

Fandiño J., Romaní-Pérez M., Outeriño-Iglesias V., Diz-Chaves Y., González-Nuñez M., Dab H.**), Vigo E., González-Matías L., Mallo F.

\section{ECE2015

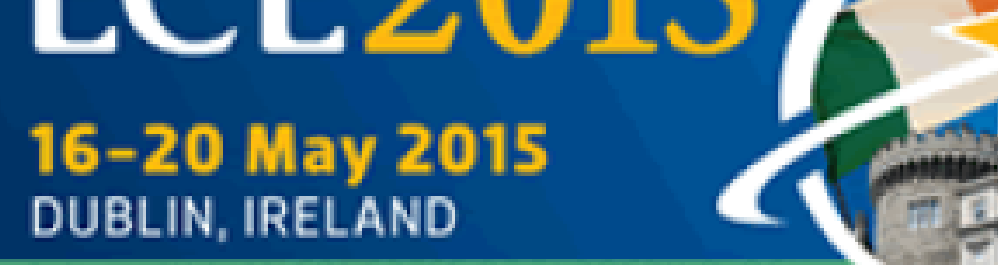

EP-620

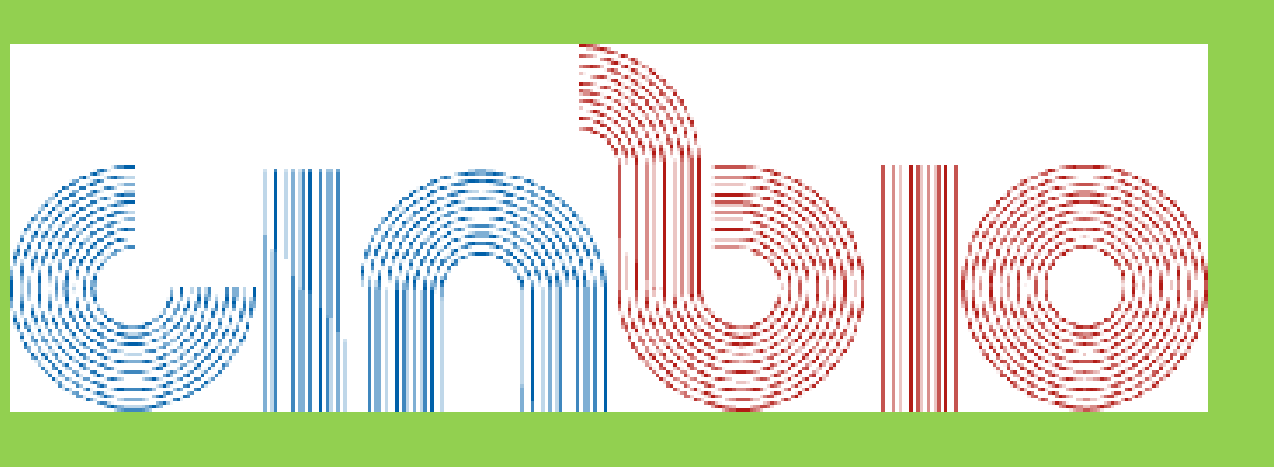

\section{Introduction}

diopathic pulmonary fibrosis (IPF) is a chronic, progressive and fatal lung disease characterized by excessive matrix deposition that disrupts the normal alveolar architecture and lung physiology. Currently, there is no effective treatment for this disease.

Glucagon-like peptide-1 (GLP-1) is a gut-produced hormone with insulinotropic effects. GLP1 receptor (GLP-1R) is widely expressed in different tissues and organs, including the lung. GLP-1 plays an essential role in the synthesis of the Surfactant Proteins (SPs), specially the SP-B produced by type II pneumocytes, crucial for the surfactant stability and maintenance of the respiratory mechanics.

In myocardial tissue, GLP-1 system can block the development of fibrosis, inhibiting the mRNA expression of pro-fibrotic cytokines and other elements of the fibrotic response (1,

Previous data from our laboratory have shown that Liraglutide administration to Streptozotocin-diabetic rats is able to reverse right ventricular hypertrophy (submitted) and to restore normal levels of the surfactant-associated proteins in an animal model of delayed lung maturation (3)

\section{Objetives}

To study the effects of administration of the GLP-1 receptor agonist Liraglutide in an animal model of idiopathic pulmonary fibrosis induced by bleomycin, during the late fibrotic phase. Specifically:

1- The expression of the fibrotic cytokine CTGF and Collagen type 1 alpha-1.

2- The myocardium masses.

3- The levels of Surfactant Protein B and his transcriptional factor TTF-1.

4- The changes in the expression levels of the receptors for metabolic hormones present in the lung.

\section{Material and methods}

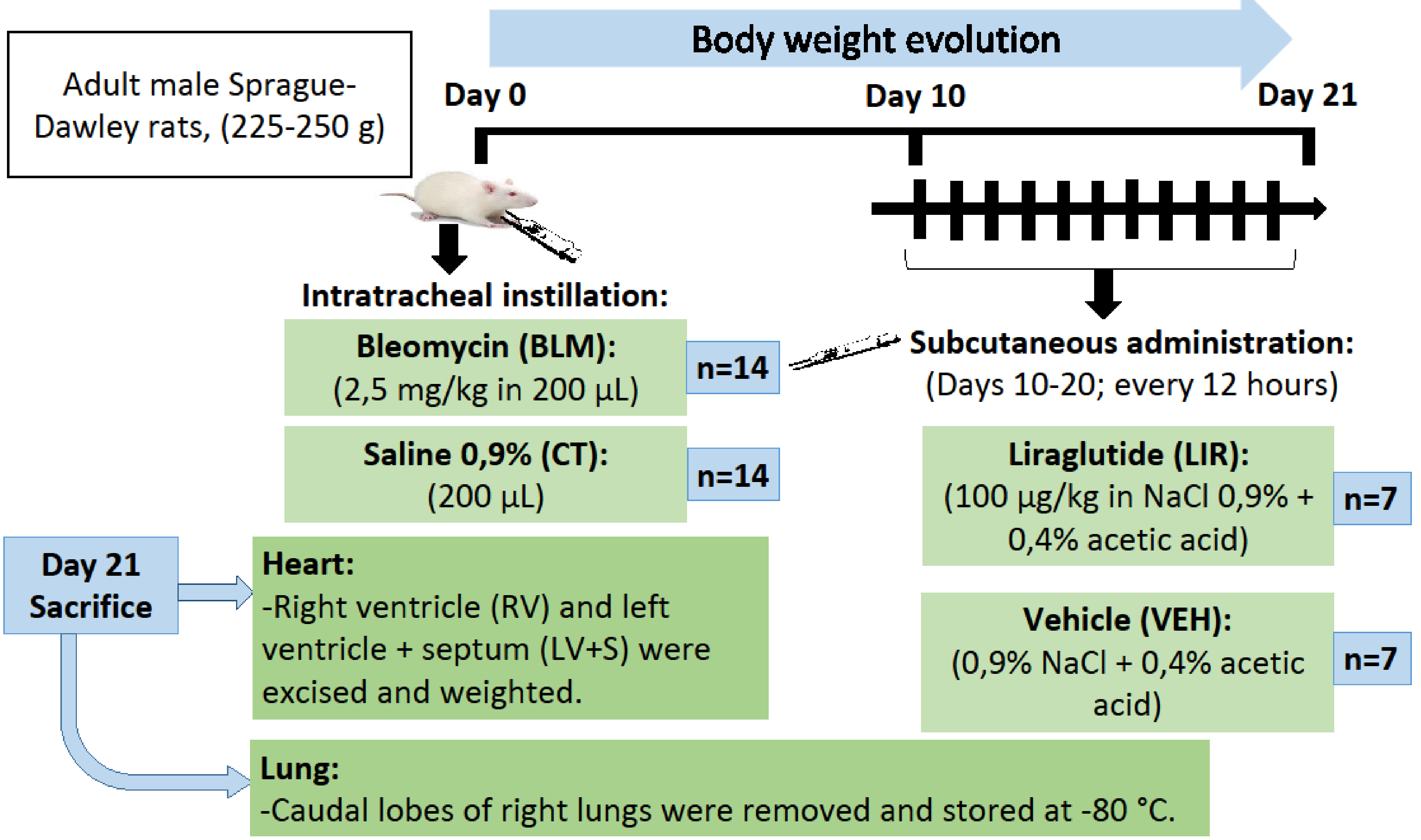

mRNA expression analysis

Extraction with Chowczinsky \& Sacchi single step method.

- Retro-transcribed to cDNA and running in a conventional RT-PCR for each signal.

\section{Statistics}

-Non parametric Kruskall-Wallis Test following by pairwise multiple comparisons.

"*" $\rightarrow$ Statistically different with CT/VEH group.

" \&" $\rightarrow$ Statistically different with CT/LIRA group.

"\#" $\rightarrow$ Statistically different wih BLM/VEH group.

$*, \&, \# \rightarrow p \leq 0,05 ; * *, \& \&, \# \# \rightarrow p \leq 0,01 ; * * *, \& \& \&, \# \# \rightarrow p \leq 0,001$

\section{$\underline{\text { Results }}$}

Effects in myocardial masses
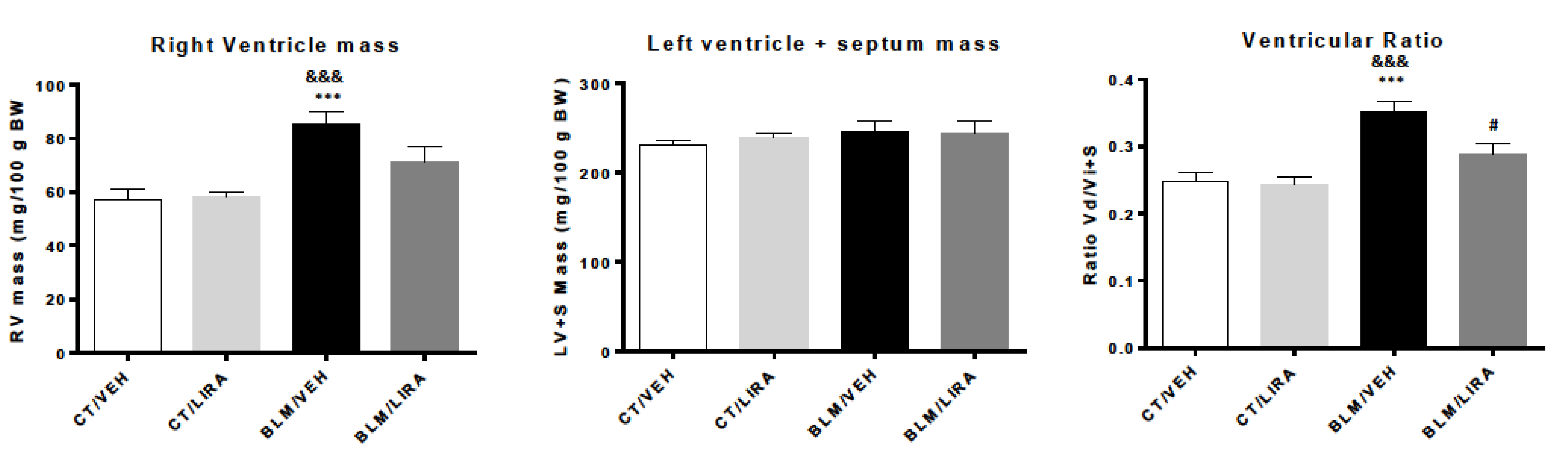

- Bleomycin administration increases ventricular ratio $\rightarrow$ suggesting pulmonary arterial hypertension.

- This increase in ventricular ratio is due to an increase in the right ventricle mass. Left ventricle mass

remain unchanged.

- Treatment with Liraglutide partially reverses the increases of ventricular ratio, observed in BLM/VEH group. This effect is due to a reduction in right ventricle hypertrophy.
Universida $\mathrm{de}^{\text {Vigo }}$

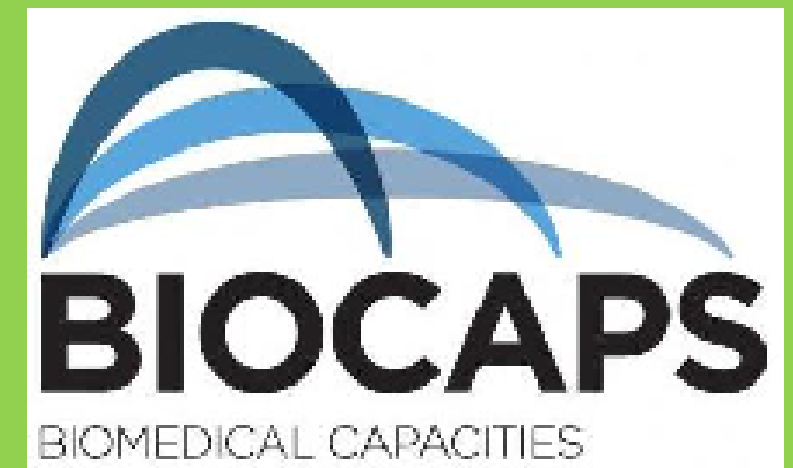

$\underline{\text { Results }}$

Effects in fibrosis indicators

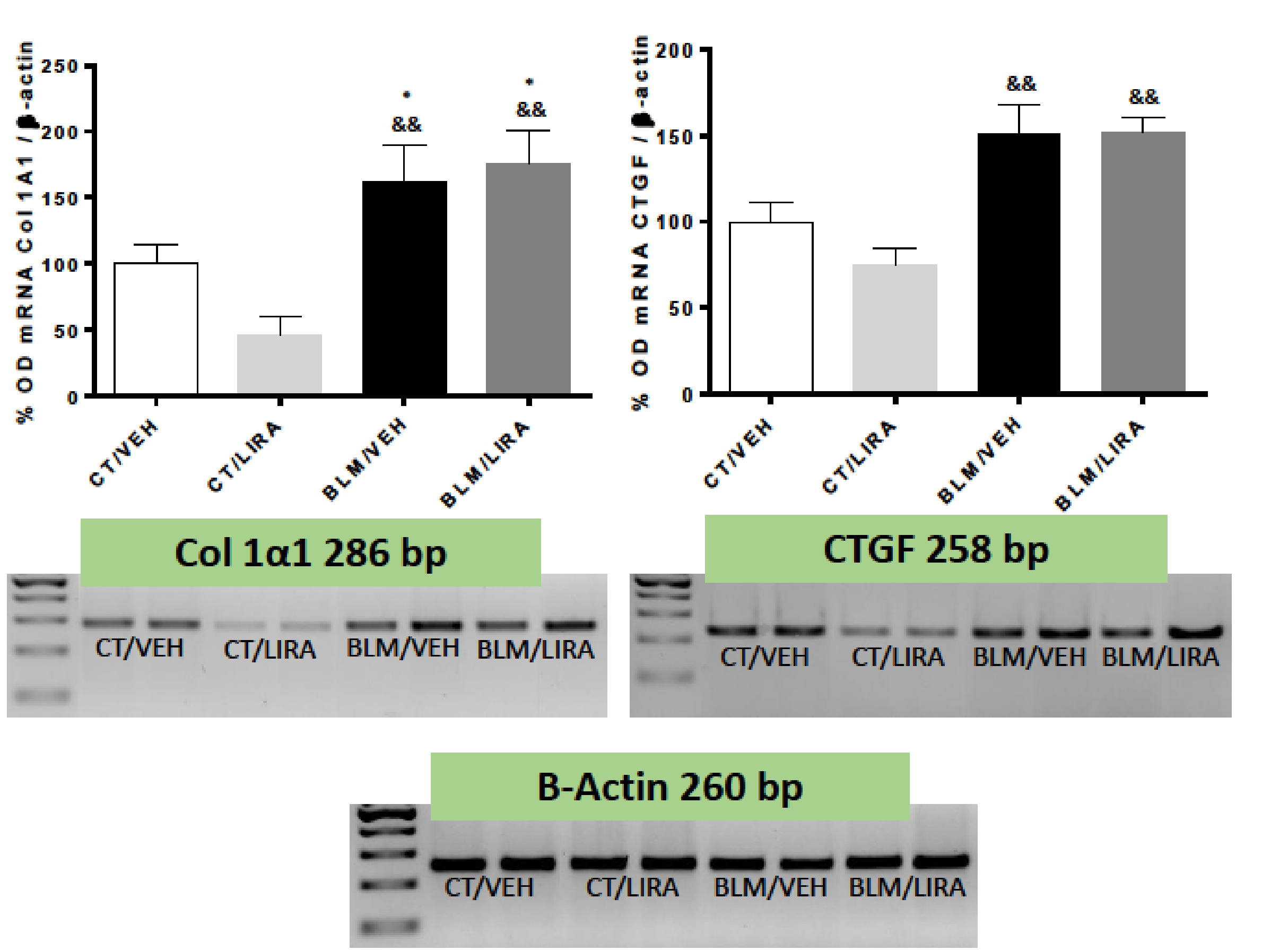

- Bleomycin administration increase mRNA expression levels of Collagen type 1 alpha 1 (Col 1 $\alpha 1)$ and Connective Tissue Growth Factor (CTGF).

No differences had been observed after 11 days treatment with liraglutide
Effects over Surfactant Protein B and his transcriptional factor TTF-1

Bleomycin administration strongly decreases the mRNA levels of Surfactant Protein B (SP-B) and his transcription factor: Thyroid Transcription Factor 1 (TTF-1).

- Liraglutide treatment completely restores mRNA of SP-B, and partially of TTF-1.
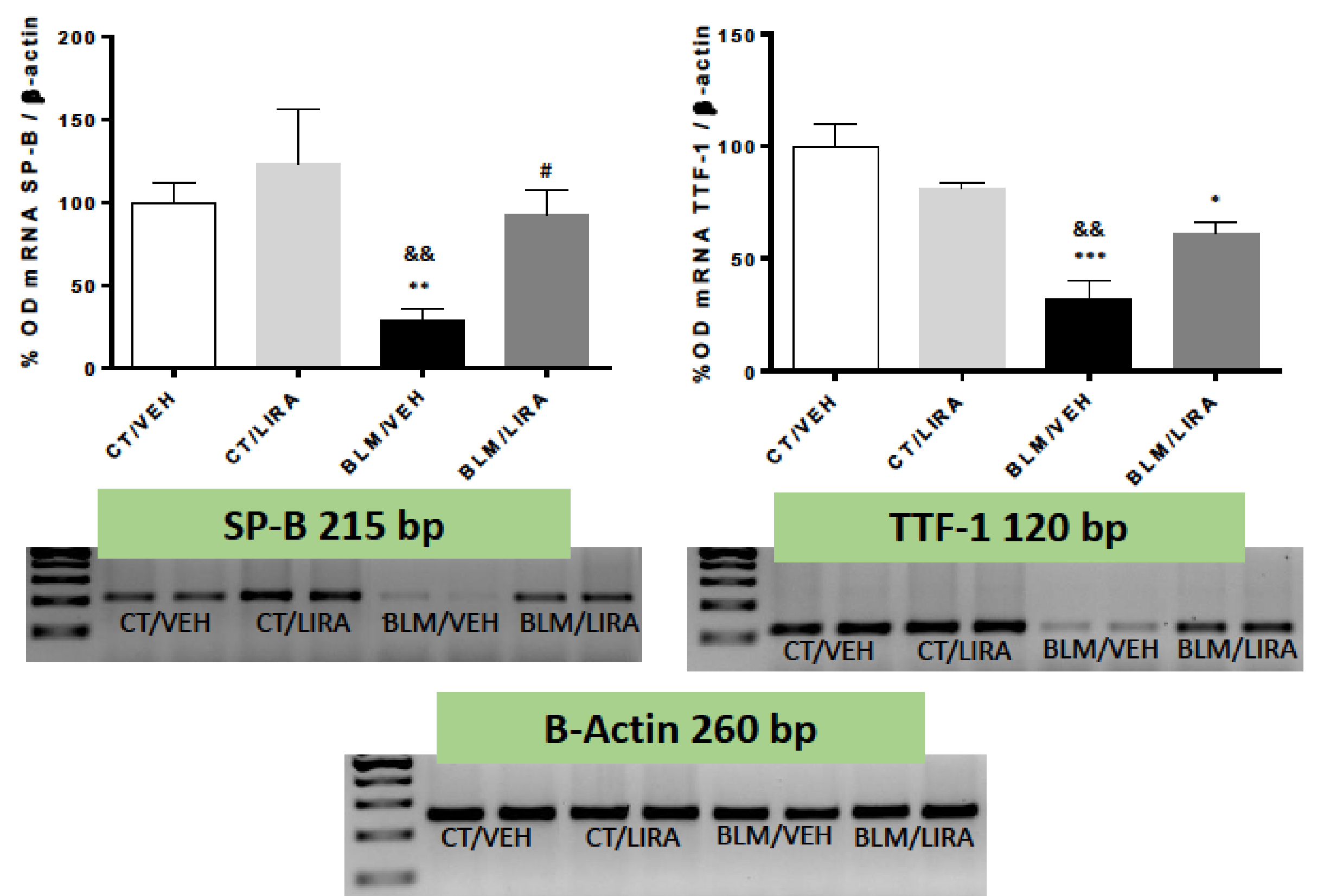

Effects in receptor of metabolic hormones: GLP-1 R and Lep-R

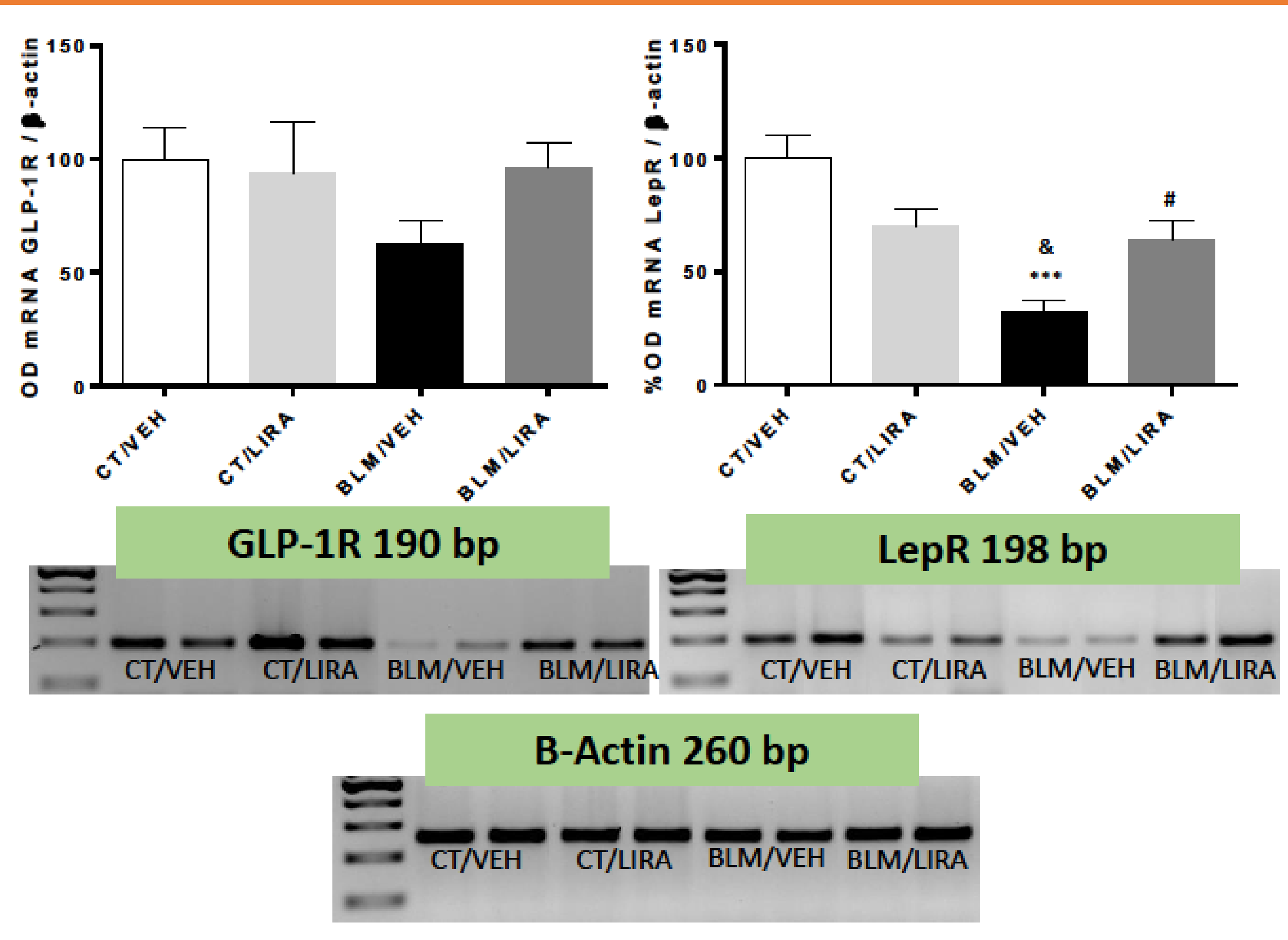

- Bleomycin administration decreases mRNA expression levels of both receptors.

- Liraglutide treatment completely restores the expression levels of GLP-1R and partially of LeptinR.

\section{Conclusion}

11 days of treatment with Liraglutide did not modify the levels of fibrosis indicators, when the fibrotic state is fully established.

$\square$ Liraglutide treatment restored Surfactant Protein B mRNA deficiency in the BLM model of lung fibrosis, such as we observed previously in an animal model of delayed lung maturation (3). This should improve alveolar function and lung mechanics in fibrosis.

$\square$ Liraglutide treatment reversed right ventricular hypertrophy, such as we previously observed in the STZ animal model of type 1 diabetes.

As a whole, Liraglutide treatment in the late fibrotic phase in the animal model of IPF, is unable to reduce the expression of fibrotic factors. Despite, there are some positive effects that contribute to a better lung and cardiopulmonary function.

\section{References}

1-Picatoste B. Ramírez E Caro-Vadillo A Iborra C Egido J, Tuñón J \& Lorenzo O. Sitagliptin reduces cardiac apoptosis, hypertrophy and fibrosis primarily by insulin-dependen mechanisms in experimental type-II diabetes. Potential roles of GLP-1 isoforms. PLOS ONE 2013 8(10) e78330.

2-Anagnostis P, Athyros VG, Adamidou F, Panagiotou A, Kita M, Karagiannis A \& Mikhailidis DP. Glucagon-like-peptide-1-based therapies and cardiovascular disease: looking beyond glycaemic control. Diabetes, Obesity and Metabolism 201113 302-312.

3-Romaní-Pérez M, Outeiriño-Iglesias V, Gil-Lozano M, González-Matías L, Mallo F \& Vigo E. Pulmonary GLP-1 receptor increase at birth and exogenous GLP-1 receptor agonists augmented surfactantprotein levels in litters from normal and Nitrofen-treated pregnant rats. Endocrinology 2013154 1144-1155. 